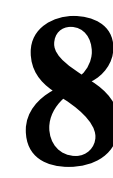

\title{
LA EDUCACIÓN FEMENINA EN LA INDIA DURANTE LA ÉPOCA COLONIAL
}

(WOMEN EDUCATION IN INDIA DURING THE COLONIAL PERIOD)

Alejandra Val Cubero

Universidad Carlos III de Madrid

\section{RESUMEN}

El sistema de educación femenina en la India es un legado del colonialismo británico puesto en funcionamiento por las misioneras y por los grupos de reformistas indios a mediados del siglo XIX. Las misioneras trataron de propagar la religión cristiana a través de la educación y los reformistas abogaron por una educación siguiendo las tradiciones indias de respeto y sumisión, donde la espiritualidad y la sensibilidad no sólo diferenciaba las mujeres indias de las que no lo eran, también a las mujeres de las castas superiores de otras castas que no podían acceder a la enseñanza formal.

Este artículo surgió durante mi estancia en la universidad de Jawahalhal Nehru en Delhi donde pude visitar instituciones educativas como el Victorian Institution. El método de investigación es el genealógico: retraernos al pasado para ver los cambios y transformaciones en los procesos sociales y educativos con el fin de comprender el presente. En la actualidad realizo un estudio sobre la educación femenina en la India en el siglo XXI.

\section{ABSTRACT}

The female education system in India is a legacy of the British colonial times, initiated by missionaries and Indian reformists in the middle of the $19^{\text {th }}$ century. While missionaries intended to spread Christianity through this education system, the reformists contributed to the design of an education system 
following the Indian tradition of respect and submission, where spirituality and sensitivity not only differentiate Indian women from those who are not, but also women of superior castes from the ones of other castes who cannot have access to formal education.

The idea of this article came to me during my stay in the Jawaharlal Nehru University in Delhi where I visited educational institutions such as the Victorian Institution. The research method chosen is the genealogical one: in order to understand the present, we portrait the past and identify changes and transformations which occurred in social and educational processes. $\mathrm{Cu}-$ rrently, I am conducting a study on women's education in the $21^{\text {st }}$ century.

\section{INTRODUCCIÓN}

Desde mediados del siglo XIX el tema de la educación femenina en occidente y también en oriente se convirtió en un asunto de debate público y fue tratado desde ópticas muy diversas. Los sectores más progresistas veían la incorporación de las mujeres a las aulas como un acto natural y necesario al que se oponían los grupos más conservadores, quienes apelaban a la tradición y a las costumbres para mantener el orden social, orden que se alteraría si las mujeres accedían a las aulas porque todo cambio traería consecuencias negativas en las aptitudes y comportamientos propiamente femeninos.

Según los discursos de médicos, psicólogos y juristas occidentales, "los hombres" y "las mujeres" eran diferentes, agudizándose la presunta inferioridad de las últimas como seres pasivos y enfermos. Para muchos médicos e intelectuales, como es el caso de Jules Michelet, cada etapa del desarrollo reproductor de las mujeres, desde la primera menstruación hasta la menopausia, constituía una especie de enfermedad que debía ser controlada y vigilada por un médico. No sólo se publicaron numerosas obras médicas centradas en este aspecto, sino también todo tipo de tratados sobre educación y moral en los que se analizaban y se establecían las diferencias de comportamiento entre los sexos. Estas diferencias en la manera de comportarse, de vestir, de hablar... derivaban de la nueva concepción del cuerpo, tanto masculino como femenino, que se fue gestando en este periodo (Laquer, 1989). Los escritos pedagógicos como los de Rousseau son también fundamentales para entender por qué la mujer debía recibir una educación distinta a la del hombre. En El Emilio escrito en 1762 no se habla de la mujer en términos de igualdad, sino como complemento del hombre. Este libro fue uno de los ensayos que más influyeron en la nueva visión de las diferencias sexuales. Según el pensador francés, la educación concernía exclusivamente al hombre, y la única forma de asegurar el orden político y social era excluir 
a las mujeres del ámbito público, pues la presencia femenina distraería a los hombres, y traería consigo la promiscuidad. También Kant describió a hombres y mujeres como poseedores de características opuestas. Tanto para Kant como para su discípulo, Fitche, el impulso sexual masculino estaba íntimamente ligado a un deseo de independencia y libertad. Sobre la idea de la pasividad sexual femenina se asentaba la creencia de que las mujeres no eran merecedoras de derechos políticos ni de la plena ciudadanía, de ahí que su ámbito de actuación se limitase a lo doméstico.

Familia, sexualidad y procreación son tres conceptos que van de la mano en todo el siglo XIX en occidente. La idea de encauzar la sexualidad hacia la monogamia y la necesidad de controlar la propia sexualidad a fin de no contraer enfermedades peligrosas fueron los dos pilares en los que se apoyó la mentalidad burguesa. Las concepciones de lo masculino y lo femenino afectaron y a su vez se vieron afectadas por desarrollos políticos o sociales concretos. A partir de la Revolución Francesa el liberalismo y el socialismo ofrecieron a "las mujeres" nuevas oportunidades de participación y de exclusión. La demanda de derechos legales y políticos, y la participación de "las mujeres" en la Revolución Francesa, vinculó los conceptos de masculinidad y ciudadanía. Esta habitual imagen masculina de "poder, posesión y dominio" estuvo ligada de manera inevitable a la "sumisión, pasividad y disponibilidad" de "la mujer". La edición de 1842 de la Enciclopaedia Britannica afirmaba rotundamente que "el hombre audaz y vigoroso está calificado para ser protector (...), y la mujer siempre desprecia al hombre que carece totalmente de estas cualidades" (Enciclopaedia Britannica, 1842).

La importancia que adquirió la sexualidad y su control en la vida burguesa en el siglo XIX está también relacionada con el intervencionismo del Estado en su afán colonialista. Las teorías de Darwin se hicieron mundialmente famosas a partir de 1860 y se aplicaron en contextos de evolución social y de diferencias de género. En esta jerarquía "la mujer" europea se situaría en una escala inferior al hombre europeo, pero en una escala superior al hombre y a la mujer "salvaje", a los que había inevitablemente que civilizar.

En la India del siglo XIX la idea de la separación de los sexos y del control de la sexualidad también fue alentada y promovida por los administradores británicos, para quienes el hogar, además de ser el espacio propiamente femenino, se había convertido en la extensión del imperio colonial que había que proteger y salvaguardar. El hogar tenía que ser un reducto de paz y tranquilidad, un lugar armonioso y puro, distinto del impuro, sucio y bullicioso espacio exterior indio. Pero no eran éstas las únicas ideas que habían llegado a la colonia: los incipientes movimientos feministas en Gran Bretaña se estaban planteando el funcionamiento de la familia, la relación 
amorosa y la maternidad con la publicación de una serie de textos claves, como La sujeción de la mujer, publicado por Stuart Mill en 1869, y El origen de la familia, la propiedad privada y el Estado, de Engels, escrito en 1884, y a los que tendrían acceso ciertos reformistas indios pertenecientes al Brahmo Samaj, un grupo fundado a mediados del siglo XIX que promulgaba, entre otros aspectos, la importancia de la educación femenina como camino hacia el desarrollo del país y la independencia con Inglaterra.

\section{LAS MISIONERAS Y LAS PRIMERAS INSTITUCIONES EDUCATIVAS}

El precepto de Charter Renewal Act de 1813 levantó las restricciones de los misioneros y misioneras que querían desplazarse a la India y a partir de esta fecha la entrada de grupos cristianos en el país se hizo más frecuente. Por lo general, las instituciones misioneras femeninas contaban con férreos estatutos de admisión, como fue el caso The Ladies Association for the Promotion of Female Education que realizaba un examen "moral" a todas sus candidatas: la edad máxima de ingreso era de treinta años, debían estar solteras, aportar la cartilla de buena salud y certificar un correcto espíritu cristiano, así como capacidad suficiente para transmitir la devoción cristiana en condiciones adversas. La institución verificaba si el compromiso cristiano era verdadero y adecuado mediante una exhaustiva entrevista sobre la vida privada de la joven que incluía el tipo de libros que leía, su participación en obras de caridad y sus aspiraciones futuras -incluidas sus inclinaciones hacia el matrimonio y la maternidad-. Durante la entrevista se valoraba la paciencia, el buen carácter y el conocimiento en materias diversas como la música, la pintura, las manualidades y la confección. Las jóvenes seleccionadas seguían un breve curso introductorio y firmaban un contrato de estancia en la India de tres años, por el que percibían un salario anual de ciento veinte libras, la mitad del sueldo que recibían sus compañeros por el mismo trabajo y que variaba según su estado civil: doscientas veinticinco libras si estaban solteros y trescientas libras si estaban casados (Forbes, 2000). The Ladies Auxiliary of the Society for the Propagation of the Gospel in Foreign Parts, creada en 1895 y dependiente de la iglesia británica, y The South Australian Baptist Missionary Society, que comenzó su andadura en el estado de Bengal en 1882, tenían estatutos similares, y ambas instituciones llegaron a tener más de doscientas misioneras trabajando en la India a finales del siglo XIX.

Las misioneras, tal y como demostró la historiadora Geraldine H. Forbes en su estudio sobre la vida de éstas mujeres en el subcontinente asiático, tenían serías dificultades para adaptarse a la vida de un país del que desconocían casi todo, aunque — como señala a autora-, si lograban sobrevivir, 
su salario comparado con el salario de una maestra en Inglaterra era más elevado y el status social de su profesión era más reconocido tanto en la India como a su vuelta a Inglaterra (Forbes, 2000). Si los británicos habían tenido contacto continuo con la población autóctona desde el siglo XVII e incluso antes, documentos sobre la vida y trabajo de las mujeres misioneras muestran cómo la relación entre las mujeres británicas y las mujeres indias era prácticamente inexistente a comienzos del siglo XIX y solo comenzó a ser habitual a partir de 1860 , cuando los primeros programas educativos femeninos fueron puestos en marcha (Forbes, 2000).

La presencia de las misioneras en la India tenía un claro contenido ideológico: educar en la fe cristiana, lo que iniciaron tímidamente dentro de las viviendas o zenanas donde impartían sus clases y talleres. Sin embargo la injerencia cada vez mayor de las misioneras en las labores educativas provocó las críticas de miembros reformistas como Swami Vivekanda, quien señaló la sumisión de los brahmanes a las peticiones inglesas; según Vivekanda, las jóvenes debían asistir a la escuela, pero de la mano de profesores nativos que enseñaran las costumbres y tradiciones autóctonas, sin someterse a ninguna injerencia exterior.

La historiadora Radha Kumar cuenta en su obra The History of Doing cómo el movimiento para la educación femenina se indianizó a lo largo del siglo XIX, pasando de cien escuelas y dos mil quinientas alumnas en 1863 a más de dos mil escuelas que acogieron un total de más de ochenta mil estudiantes en 1890 (Chatterjee, 1996). Incremento estimable y progresivo en términos absolutos aunque no alcanzara, a comienzos del siglo XX, al 2\% de la población femenina, dato que representaba a una pequeña élite culta, concentrada fundamentalmente en las zonas urbanas del estado de Bengal (Southard, 1993). En 1800 no había ni una sola escuela para niñas y la primera escuela nativa, The London Missionary Society abrió sus puertas en 1818 , justo un siglo antes de que se inaugurase la primera universidad médica para mujeres, The Lady Hardinge Medical College, fundada Delhi en 1916 (Lind, 1988).

\section{EDUCAR PARA UNA MINORÍA: LA NUEVA BHADRAMAHILA Y LA “SUPERIORIDAD ESPIRITUAL" DE LA MUJER INDIA}

Hasta mediados del siglo XIX la educación femenina de las castas superiores consistía en memorizar y en leer textos sagrados para ser recitados en ceremonias y fiestas familiares. La emigración de la élite india de las zonas rurales a las zonas urbanas, y su incipiente participación en la administración británica modificaron ciertas costumbres y crearon nuevos estilos de vida: mientras que "los hombres" pasaban más tiempo fuera del hogar, 
adaptándose a los nuevos usos ingleses -formas de vestir, de comer, de relacionarse ...- "las mujeres" de las castas superiores instaladas en las zonas urbanas pasaron a ser las depositarías de ciertas tradiciones, como las celebraciones religiosas o pujas.

Para aquellos que trabajaban en la administración británica, la educación formal de sus esposas o hijas comenzó a ser no sólo aceptable sino que se convirtió en un requisito fundamental de la nueva bhadramahila o mujer respetable -imagen que tendría su correspondencia en el prototipo victoriano de la mujer de clase media-. Educar a los miembros femeninos de las castas consideradas superiores ayudaba a que la familia ascendiera en la escala social a través de los enlaces matrimoniales. Ya no era incompatible tener nociones de literatura o arte y ser una mujer honesta y culta, lo que había que evitar a toda costa era convertirse en una memsahib, en una extranjera con hábitos y costumbres ajenas y nocivas. Educar sí, pero siguiendo las tradiciones indias de respeto y sumisión al esposo y a la comunidad.

El impulso de la educación se convirtió en un arma de lucha nacional, según los reformistas indios la educación de "las mujeres indias" las situaba en un grado superior a las mujeres occidentales, porque si para éstas -las occidentales- la educación consistía en adquirir habilidades materiales para competir con los hombres en el mundo exterior, perdiendo las virtudes propiamente femeninas, "las mujeres indias" fundamentaban su educación sobre los valores espirituales. La espiritualidad y sensibilidad no sólo diferenciaba a "las mujeres indias" de las que no lo eran, también a las mujeres de clase alta de otros grupos que no podían acceder a la enseñanza formal (Chatterjee, 1996). Siguiendo esta pauta, aspectos como la "disciplina" y el "orden" fueron claves para dar forma a las fantasías nacionalistas. Los británicos eran poderosos - argumentaban- porque eran disciplinados, ordenados y puntuales en todos y cada uno de los detalles de sus vidas y esto era posible gracias a la educación de sus mujeres, quienes aportaban al hogar las virtudes de orden y disciplina. El impulso de la educación femenina a comienzos del siglo XX se convirtió en un arma ideológico para mostrar a los británicos que ellos también podían educar sin necesidad de su presencia y que además el tipo de educación que recibían sus mujeres era mucho mejor.

A partir de este momento, el tema de debate ya no se centró en la importancia de la educación femenina sino en el tipo de educación que debía impartirse y hasta qué edad, aspectos que provocaron las luchas y disputas entre los sectores indios más tradicionales y los más progresistas; el sector más liberal abogaba por una escuela mixta y en 1876 creó el Bengal Women's Collegue, mientras que el ala más tradicional defendió un sistema curricular diferente para cada sexo y fundó The Victorian Institution, que en 1911 se convirtió en el Victoria Collegue. Tanto el Bengal Collegue como el Victorian 
Institution pasaron a convertirse en los centros de élite de las jóvenes de las castas de los brahmanes, jóvenes que pasarían a encabezar los movimientos de mujeres a principios del siglo XX; allí se formó la escritora y trabajadora social Pandita Ramabai, directora de la Arya Mahila Samaj en Poona, quien abogó por la integración de niños y niñas en la escuela para contrarrestar los efectos del purdah o la reclusión de las jóvenes dentro del hogar y sería luego uno de los apoyos incondicionales del movimiento de no-violencia ideado por Mahatma Gandhi.

Otro de los temas de discusión pública fue el papel de la lengua inglesa en el sistema curricular. El inglés era la lengua oficial del gobierno y de la administración desde 1835 y su dominio era clave para acceder a los trabajos mejor remunerados y con mayores posibilidades de ascenso social, pero al no estar la educación femenina encaminada a dotar a las mujeres de conocimientos y habilidades para ser utilizados en el ámbito laboral, sino para hacer de ellas mejores madres, esposas e hijas, la enseñanza del inglés se restringió a unos cuantos colegios de élite. La directora y fundadora del Sakhawat Girls' School, R.S Hossein, expuso de manera clara el sentido de la educación femenina y su utilidad: "India debe conservar los mejores elementos de su periodo dorado incorporando lo que es útil de la tradición occidental (...) en un plazo de tiempo no muy lejano, nuestras hijas obtendrán títulos universitarios convirtiéndose en ideales hijas, esposas y madres, o mejor debería decir, obedientes hijas, amantes hermanas, respetuosas esposas y educadas madres" (La Tribune,1894).

Otro aspecto que generó continuos debates fue el tema de la coeducación; en la sesión de la Education Comisión de 1882 se decretó que el currículum debía diferenciarse en razón del sexo y que en el caso de las escuelas de niñas, las materias debían incidir en temas como la higiene, la música, el hogar, la costura y el bordado. Si en 1902 el 44,7\% de las jóvenes matriculadas estudiaban en instituciones mixtas, esta cifra descendió hasta el 38.5\%" en 1927; estos veinte años habían sido suficientes para poner en funcionamiento escuelas exclusivas para niñas y jóvenes y separarlos por sexo (Chapnick y Seymour, 1994). La educación primaria seguía siendo un asunto de élites.

\section{EDUCAR PARA UNA MAYORÍA: PERIYAR Y EL MOVIMIENTO DE AUTO RESPETO}

La mayoría de los reformistas indios que apoyaron la educación femenina abogaron por la educación de "las mujeres" de su misma condición social, aunque rara vez defendieron la promulgación de una educación universal que permitiera a "las mujeres" indias ejercer profesiones fuera del ám- 
bito del hogar. La pertenencia a una determinada clase y casta estaba todavía muy presente en sus discursos y afirmaciones.

Uno de los movimientos que desde la segunda década del siglo XX comenzó a manifestarse sobre la importancia de la emancipación de las mujeres sin diferencia de clase, casta y religión, fue el movimiento de Suyamariathai Iyakkkam o Movimiento de Auto Respeto, creado por Periyar E. V Ramasamy en 1926. Periyar nació en el seno de una familia adinerada, aunque desde temprana edad pudo ver las fuertes discriminaciones sociales y económicas que sufría la gran mayoría de la población por razones de casta, etnia, clase social y género, lo que motivó su implicación social y política, primero de la mano del Indian National Congress en 1919, militancia que abandonaría en 1925 al no compartir con los presupuestos elitistas del partido y más tarde formando su propio grupo político. Entre los años 1929 a 1932 recorrió Malasia, Europa y Rusia, viajes que tuvieron una influencia en su formación intelectual y en 1939 fundó el Justice Party, que pasaría a llamarse Dravidar Kazhagam en 1944.

El líder político al contrario que la mayoría de los reformistas bengalíes creía que la ruptura con las tradiciones era la única manera de instaurar el progreso. De esta manera lo expuso en el South Indian Reform Conference de 1928: "He perdido la fe en las reformas sociales. Para alguien que cree en los cambios radicales basados en el respeto, igualdad, progreso, la alternativa a la situación actual no es la mera reforma, sino un trabajo de reconstrucción radical que debería pasar por destruir las estructuras tradicionales" (Kudi Arasu, 1928). Para Periyar la educación femenina no consistía en formar buenas madres y esposas sumisas; en sus escritos señaló y criticó una y otra vez las desigualdades entre los sexos y entre las diferentes castas. En uno de sus artículos, Why Did Women Become Slaved? - ¿Por qué las mujeres se han convertido en esclavas?-, abogó por el divorcio, criticó los matrimonios concertados y no deseados e incluso tildó de sexista su propio idioma materno - el tamil — porque no existían sinónimos para todas las palabras, como era el caso de viudedad y adulterio que sólo existía en su versión femenina, inventando el neologismo de vidavan para los viudos y vibacharan para los hombres que ejercían la prostitución.

El movimiento de auto respeto promovió los matrimonios entre diferentes castas y religiones; impulsó las bodas con viudas, alianzas que ponían en tela de juicio las tradiciones hindúes más enraizadas porque tenían lugar sin la presencia de un brahmán y durante la noche - momento nada apropiado según la creencia hinduista-. Entre 1929 y 1932 se estimó que más de 8.000 matrimonios de este tipo habían tenido lugar en el estado Tamil Nadu, matrimonios que se siguen realizando hoy en día (Kudi Arasu, 1948). 


\section{A MODO DE CONCLUSIÓN}

Desde mediados del siglo XIX los misioneros ingleses tuvieron un papel destacado en el análisis de la sociedad india en general y de "la mujer india" en particular. El reverendo El Storrow llegó a la India en 1848 y participó del discurso imperialista a través de sus escritos y conferencias públicas. Para Storrow los países históricamente poderosos como la Roma antigua o la moderna Inglaterra debían su superioridad, fuerza, coraje y virtud a la posición y al respeto que otorgaban a sus mujeres; la posición de la mujer - según sus propias palabras-era un indicador excelente para medir el avance de la sociedad. Siguiendo el mismo discurso que James Mill en History of British India, Storrow puntualizó que la sociedad india era "inferior" a otras sociedades como la inglesa por el desprecio de los hombres hacia las mujeres al permitir los matrimonios a temprana edad, su reclusión en el hogar — purdah-y la segregación y humillación de las viudas, quienes tenían prohibido volver a casarse. Otro de los teóricos conocidos y respetados entre los círculos misioneros, Charles Grant, habló sobre las costumbres indias en su obra Observations on the State of Society among the Asiatic Subjects of Great Britain, escrita en 1792, añadiendo mientras que los hombres no tienen restricciones morales y viven con la insensibilidad de los brutos, las mujeres indias tienen una vida de servidumbre, en estado de perpetuo sometimiento y una violenta y prematura muerte (Taneja, 2005).

En los decretos aprobados por los británicos entre 1795 y 1930, sus prioridades se concentraron en la ley de la prohibición del sati en 1829 - práctica considerada un suicidio encubierto porque la mujer se autoinmolaba junto al cuerpo de su marido-; la ley que permitía el matrimonio para las viudas en 1856; la ley que establecía la edad mínima para casarse en doce años en 1891; la prohibición del infanticidio femenino en 1795, 1804 y 1870 y la abolición del matrimonio entre niños en 1929. Todas estas leyes fueron aprobadas por los gobernadores británicos y obtuvieron el apoyo de ciertos reformadores indios como Raja Rammohun Roy, quien impulsó la campaña contra el sati en 1818, e Ishwar Chandra Vidyasagar, defensor del matrimonio de las viudas.

La única salvación para un pueblo incivilizado y salvaje era extender la religión cristiana y ayudar a los más desprotegidos, entre los que se encontraban "las mujeres indias". Los británicos apoyándose en el nuevo marco jurídico recientemente establecido aprobaron varios decretos con el fin de mejorar la situación de "las mujeres", sin hacer ninguna distinción de clase social, casta, lengua, etnia o hábitat.

El sistema de educación en la India es un legado del colonialismo británico, método puesto en funcionamiento por las misioneras y por los gru- 
pos de reformistas indios para quienes la educación era imprescindible si permitía a "las mujeres" desempeñar mejor ciertas cualidades y aptitudes supuestamente ligadas a su condición femenina. El tema de la educación fue utilizado como arma de doble filo dentro de los sectores reformistas y nacionalistas: por una parte, el país necesitaba mujeres educadas que participaran en su lucha nacional y, por otra, había que mostrar a los británicos que las mujeres indias eran superiores en el terreno espiritual y moral a las mujeres occidentales, rebatiendo el discurso colonial e imperialista que atestiguaba las duras condiciones que sufrían las mujeres indias, condiciones que hacía indispensable la permanencia británica.

El incremento del número de niñas que accedieron a las aulas a finales del siglo XIX y comienzos del siglo XX fue lento y muy fragmentado según las regiones, la casta y la religión a la que pertenecieran las jóvenes. Mientras que los sectores reformistas indios apoyaron el slogan que se hizo famoso en los años veinte: educar a una hija, es educar a una familia, la mayoría de la población india afincada en las zonas rurales tenía problemas más acuciantes que atender. Para ellos, educar a una hija era como regar la flor del vecino, según el dicho popular que hoy todavía se escucha y que hace que el infanticidio femenino sea uno de los aspectos más discriminatorios entre hombres y mujeres. La "nueva mujer" que accedía a la formación reglada, aprendía idiomas y tenía la posibilidad de viajar dentro y fuera del país formaba parte a una minoritaria élite urbana cuyos padres y hermanos habían recibido una educación siguiendo el sistema curricular británico y ocupaban altos cargos en la administración colonial. Muchas de ellas eran hijas y esposas de miembros de grupos reformistas como es el caso de Mrinalini Sen o Swarnakumari Devi, ambas reconocidas novelistas y editoras.

Sin embargo, fueron las primeras generaciones de mujeres educadas en colegios de élite como el Victorian Institution o el Bengal Collegue quienes no se conformarían con ser buenas y sumisas esposas e inaugurarían asociaciones como The Women's Indian Association, The National Council of Women in India y The All-Indian Women's Conference que nacieron entre 1917 y 1927 y abogaron por el voto femenino, abriendo nuevos caminos y extendiendo la educación femenina a otras castas más desprotegidas.

La tímida apertura económica que comenzó a finales de los años ochenta ha repercutido en las políticas educativas. La aprobación del New Education Policy en 1986, incrementó considerablemente el presupuesto en educación. Esta nueva medida junto con la formación de una más que respetable clase media, que según los analistas cuenta con más de trescientos cincuenta millones de personas - en un país que supera el billón de habitantes - han dado lugar a la creación de escuelas y universidades en todos los estados. Sin embargo, el absentismo escolar entre los miembros de 
las castas y clases más desfavorecidas sigue siendo considerable y reproduce las diferencias entre los más ricos y pobres. El recién nombrado nuevo gobierno, liderado por Manmohan Sing del Partido en el Congreso deberá tener como una de sus prioridades la puesta en práctica de una escuela laica y de calidad en un momento en el que crecen las tensiones y la violencia entre las diferentes religiones y etnias. 


\section{REFERENCIAS BIBLIOGRÁFICAS}

Borthwick, M. (1984). The Changing Role of Women in Bengal 1849-1905. Princenton: Princenton University Press.

Burton, A. (1996). Contesting the Zenana: The Mission to Make "Lady Doctors for India' 1874-1885. The Journal of British Studies, 35 (3), 363-397.

Chapnick, C. y Seymour, S. (1994). Women, Education and Family Structure in India. London: Westview Press.

Chatterjee, P. (1996). Colonialismo, nacionalismo y mujeres colonizadas: el debate de la India. Arenal, 3 (2), 177198.

Forbes, G. (2000). In Search of the Pure Heathen: Missionary Women in Nineteenth Century India, en Ideals, Images and Real Lives: Women in Literature and History, Bombay: Orient Longman, 68-90.

Halder, S. (2009). Prospects of higher education of the challenged women in India. International. Journal of Inclusive Education, 13 (6), 633 -646.

Laqueur, Th. (1989). Bodies, Details, and the Humanitarian Narrative, en The New Cultural History, Berkeley, LA: University of California Press.
Lind M. A. (1988). The Compassionate Memsahibs, Welfare Activities of British Women in India, 1900-1947. Connecticut: Greenwood Press.

Ray, B. (1991). Women of Bengal. Transformation in Ideas and Ideals. 19001997. Social Scientist, 19 (5/6), 3-23.

Sankar, D. (2007). What is the progress in elementary education participation in India during the last two decades. The World Bank. Disponible en: http://siteresources.worldbank.org/INT INDIA/21328531191444019328/ 21497941/SankarProgressinElementaryEducationusingNSS.pdf [Consulta: 2009, 10 de enero ].

Southard, B. (1993). Colonial Politics and Women's Rights: Women Suffrage Campaigns in Bengal, British India, in the 1920s. Modern Asian Studies, 2 (2), 397-439.

Srivastava, G. (2005). Women's education in India: issues and dimensions. Delhi: Academic Excelence.

Tobin, M. (2008). A sociological study of women's educational networks in India. Lewiston, N.Y: Edwin Mellen Press. 


\section{PALABRAS CLAVE}

India, género, educación, colonización.

\section{KEY WORDS}

India, gender, education, colonization.

\section{PERFIL ACADÉMICO DE LA AUTORA}

Alejandra Val Cubero es profesora en el Departamento de Periodismo y Comunicación Audiovisual de la Universidad Carlos III de Madrid. La autora ha realizado sucesivas investigaciones postdoctorales en Harvard (03) y la universidad de Jahawarlal Nehru University en Delhi (05), especializándose en temas relacionados con el género, la educación y los medios de comunicación. Durante el año 2006 trabajó en la consultora Altai Research en Kabul (Afganistán), donde colaboró en proyectos para organizaciones internacionales como el Banco Mundial y USAID.

Dirección de la Autora Facultad de Humanidades

Comunicación y Documentación.

Edificio Ortega y Gasset.

C/ Madrid, 133

(28903) Getafe (Madrid) España

E-mail: Alejandra.val@uc3m.es

Fecha de Recepción del artículo: 16. Septiembre. 2009

Fecha de Revisión del artículo: 12. Enero. 2010

Fecha de Aprobación del artículo: 13. Enero. 2010 